\title{
In vitro water resistance evaluation of a bioactive sunscreen containing distinct film / barrier-forming agents
}

\author{
Avaliação in vitro da resistência à água de um fotoprotetor bioativo contendo diferentes filmes / \\ agentes formadores de barreira
}

\author{
Chin Yi Su${ }^{1}$, Ana Lucía Morocho-Jácome ${ }^{1}$, Fabiana Vieira Lima ${ }^{1,2}$, Gabriela Argollo Marques ${ }^{1}$, Wendi Lucia \\ Avila Rodriguez ${ }^{1}$, Claudia Alejandra Cruz Julca ${ }^{1}$, Esther del Rocio Abanto Robles ${ }^{1}$, Catarina Rosado ${ }^{3}$, Maria \\ Valéria Robles Velasco ${ }^{1}$, André Rolim Baby ${ }^{1}$
}

\author{
${ }^{1}$ Department of Pharmacy, Faculty of Pharmaceutical Sciences, University of São Paulo, São Paulo, Brazil \\ ${ }^{2}$ Health Sciences Department, Faculty of Pharmacy, Federal University of Espírito Santo, Brazil \\ ${ }^{3} \mathrm{CBIOS}$ - Universidade Lusófona's Research Center for Biosciences and Health Technologies, Lisbon, Portugal \\ *corresponding author: andrerb@usp.br
}

\begin{abstract}
The use of functional bioactive ingredients and the property of water resistance are differentials in the choice of more effective and safer sunscreens. Water-resistance tests are expensive, time-consuming, and usually performed on subjects that expose them to irradiation and long immersion times. Thus, the study of in vitro water resistance using different film/ barrier-forming agents is relevant for obtaining sunscreen resistant to rinsing. We aimed to evaluate the water resistance of a rutin-based bioactive sunscreen containing distinct film/barrier-forming agents by an in vitro method. The in vitro water resistance assessment (\% WRR) was carried out in a water bath. In vitro sun protection factor (SPF), critical $\lambda$ $(\mathrm{nm}), \mathrm{UVA} / \mathrm{UVB}$ ratio, and UVA protection factor (UVA-PF $(0))$ were evaluated before and after rinsing using a diffuse reflectance spectrophotometer with integration sphere. The formulation with dimethicone showed higher values of SPF after rinsing and achieved the requirement of $\%$ WRR greater than $50 \%$. All formulations showed similar variations for all other parameters. The results highlighted the property of water resistance provided by the dimethicone, indicating that this emollient is an interesting ingredient choice for sunscreens.
\end{abstract}

Keywords: Bioactive photoprotection, water resistance, film/barrier formation

\section{Resumo}

$\mathrm{O}$ uso de ingredientes bioativos multifuncionais e a propriedade de resistência à água são diferenciais na escolha de protetores solares mais seguros e eficazes. Os testes de resistência à água são caros, demorados e são realizados geralmente em sujeitos, que os expõem às irradiações e longos tempos de imersão. Assim, o estudo de resistência à água in vitro, empregando agentes formadores de filme/barreira distintos, mostra-se relevante para a obtenção de fotoprotetores resistentes à lavagem. O presente trabalho teve como objetivo avaliar a resistência à água, por método in vitro, de protetores solares bioativos contendo agentes formadores de filme/barreira. A avaliação da resistência à água in vitro (\%WRR) foi realizada em banho maria. Foram avaliados: FPS in vitro, $\lambda$ crítico, razão UVA/UVB e FP-UVA (0), antes e após a lavagem, por meio de espectrofotômetro de reflectância difusa com esfera de integração. A formulação com dimeticone apresentou maiores valores de FPS pós-enxágue e atendeu ao requisito de \%WRR maior que $50 \%$. Todas as formulações apresentaram variações similares nos demais parâmetros avaliados. Os resultados evidenciaram a propriedade de resistência à água proporcionada pelo dimeticone ao fotoprotetor, o que não ocorreu com os demais formadores de filme/barreira.

Palavras-chave: Fotoprotetores bioativos, resistência à água, formadores de filme/barreira 


\section{Introduction}

Exposure to ultraviolet (UV) radiation without the sunscreens can produce inflammation, DNA damage, histological changes in the epidermis, premature aging, and carcinogenesis (1). Although the use of traditional sunscreens is the primary means used to protect against UV radiation, it is insufficient to protect against damage caused by free radicals (2). Thus, there is an increasing trend in the development of sunscreens based on two aspects: first, the use of oral formulations as a complementary form of sun protection, and, second, the addition of new ingredients of natural origin in the sunscreens to increase their sun protection factor (SPF) (3).

The antioxidant compounds used in sunscreens allow the development of multifunctional formulations: while the UV filters act on the skin surface, absorbing and/ or reflecting UV, the antioxidants act on the surface (absorbing UV radiation) and/or in the deeper layers of the skin to neutralize oxidative effects $(4,5)$.

Flavonoids are phenolic compounds known for anti-inflammatory and antioxidant activities and their capability to absorb some UV radiation (6). Rutin is among the flavonoids studied in sunscreen formulations. Several studies have demonstrated that, due to its antioxidant power, rutin is capable of increasing the photoprotective performance of formulations when associated with UV filters $(5,7,8)$.

Important aspects of sunscreen are effectiveness, safety, photostability, and resistance to water removal from the skin surface (9). Water resistance indicates how much a product is capable of maintaining its SPF after immersion in a bath for a certain period of time (10).

Generally, in order to obtain sunscreens with resistance to rinsing, it is necessary to add ingredients that guarantee the good adhesion of the formulation over the skin during immersion in water (11), i.e., ingredients that have substantivity to skin surfaces. These compounds can be hydrophobic substances and/or film-forming agents (12-14). Table 1 shows examples of substances used to increase the water resistance of sunscreens according to their mechanism of action.

\section{Introdução}

A exposição prolongada à radiação UV sem uso de fotoprotetores pode provocar inflamação, danos ao DNA, alterações histológicas na epiderme, envelhecimento precoce e carcinogênese (1). Apesar do uso de fotoprotetores tradicionais ser o principal meio utilizado na proteção contra os raios UV, este se mostra insuficiente na proteção contra os danos causados pelos radicais livres (2). Assim, cada vez mais há tendência no desenvolvimento de fotoproteção baseando-se em dois aspectos: em primeiro lugar, o uso de formulações orais como forma complementar da proteção solar e, em segundo lugar, a adição de novos ingredientes de origem natural nos fotoprotetores para incremento de FPS (3).

A combinação de compostos antioxidantes com filtros solares demonstra-se promissora no desenvolvimento de fotoprotetores multifuncionais, uma vez que ambos agem sinergicamente por mecanismos diferentes: enquanto os filtros agem na superfície cutânea, absorvendo e/ou refletindo as radiações UV, os antioxidantes agem na superfície (absorvendo a radiação UV) e/ou nas camadas mais profundas da pele (neutralizando os efeitos oxidativos) $(4,5)$.

Os compostos fenólicos, como os flavonoides são conhecidos pelas suas atividades anti-inflamatórias, antioxidantes e capacidade de absorver radiação UV (6). Dentre os flavonoides muito utilizados em formulações fotoprotetoras tem-se a rutina, vários trabalhos demonstram que, a rutina, devido ao seu poder antioxidante, é capaz de aumentar o desempenho fotoprotetor de formulações quando associada à filtros UV $(5,7,8)$.

Dentre os fatores determinantes para alcançar a eficácia de um fotoprotetor estão a absorção da radiação UV, fotoestabilidade e resistência à remoção por água da superfície cutânea (9). A resistência à água indica o quanto um produto é capaz de manter seu fator de proteção solar (FPS) após imersão em banho por determinado período de tempo (10).

Geralmente, para obtenção de fotoprotetores resistentes à lavagem é necessária a adição de ingredientes que garantem a boa adesão da formulação na pele durante a imersão em água (11). Esses compostos podem ser substâncias hidrofóbicas e/ou agentes filmógenos/ formadores de filme $(12,13,14)$. A Tabela 1 apresenta exemplos de substâncias utilizadas para aumentar a resistência à água dos protetores solares de acordo com o modo de ação. 
Table 1 - Ingredients that provide water resistance according to literature. PVP = polyvinylpyrrolidone / Tabela 1 - Compostos que conferem resistência à água de acordo com a literatura. $\mathrm{PVP}=$ polivinilpirrolidona

\begin{tabular}{l|l}
\hline Film-forming / Formadores de filme & Hydrophobic barrier / Barreira hidrofóbica \\
\hline Hexadecene PVP copolymer (14)/ & Cetil dimethicona (13)/ \\
Copolímero PVP hexadeceno (14) & Cetil dimeticona (13) \\
\hline Eicosane PVP copolymer (14)/ & Lanolin (10)/ \\
Copolímero PVP eicosano (14) & Lanolina (10) \\
\hline Triacontanyl PVP copolymer (12,14)/ & Silicone oils $(12,17,28) /$ \\
Copolímero PVP tricotanil (12,14) & Óleo de Silicone(12,17,28) \\
\hline Acrylates $\mathrm{C}_{12-22}$ alkylmethacrylate copolymers (12)/ & Mineral oil $(17) /$ \\
Copolímeros acrilatos $\mathrm{C}_{12-22}$ alquilmetacrilato (12) & Óleo mineral (17) \\
\hline Polyethylene and $\mathrm{C}_{20-40}$ alcohols (18)/ & Isopropyl myristate (17)/ \\
Polietileno e alcóois $\mathrm{C}_{20-40}(18)$ & Miristato de isopropila (17) \\
\hline Silicones $(12,28) /$ & Silicones $(12,28) /$ \\
Silicones $(12,28)$ & Silicones $(12,28)$
\end{tabular}

The mechanism of action of film-forming agents in water-resistant sunscreens occurs by maintaining the filters on the skin for a longer time, preventing its removal by water alone (14). The presence of filters inside the film can also reduce the degree of transdermal penetration of these active ingredients, a factor that allows longer-lasting protection compared to traditional sunscreens, in addition to reducing the risks of exposure and the accumulation of these compounds in the body $(12,15,16)$.

Hydrophobic substances are able of forming occlusive barriers, as they are over and/or interspersed among the corneocytes, forming a diffuse and hydrophobic impermeable (or nearly impermeable) surface (17). An efficiently formed hydrophobic barrier guarantees good water resistance and can increase the SPF. Due to this property, fewer active ingredients that can be irritating to the skin might be used in the formulation (18).

The water resistance test indicates how much a product is able of maintaining its SPF after immersing the subject in a bath (using baths, pools, spa-pools, or Jacuzzi ${ }^{\circledR}$ ) with water circulation for a predetermined time followed by a drying period without the use of towels. The number of immersion and drying cycles is repeated depending on the claim of water resistance required by the product, which can be considered as "water resistant" (40 minutes) or "very water resistant" (80 minutes). The percentage of water resistance retention (\% WRR) is determined by measuring the SPF before and after immersion $(10,12,19)$.
O mecanismo de ação dos agentes filmógenos em fotoprotetores resistentes à água ocorre pela fixação dos filtros na pele, impedindo sua remoção pela água (14). A presença de filtros no interior do filme diminui o grau de penetração transdérmica de filtros, fator que permite proteção mais duradoura em comparação aos fotoprotetores tradicionais, além de diminuir os riscos de exposição e acúmulo desses compostos no organismo $(12,15,16)$.

As substâncias hidrófobas são capazes de formar barreiras oclusivas, pois se intercalam entre os corneócitos, formando uma superfície impermeável difusa e hidrofóbica (17). A barreira hidrofóbica, formada de maneira eficiente, garante boa resistência à água e aumento de FPS. Devido à essa propriedade, pode-se empregar menor quantidade de ingredientes ativos na formulação que podem ser irritantes à pele (18).

$\mathrm{O}$ teste de resistência à água indica o quanto um produto é capaz de manter o seu fator de fotoproteção solar (FPS) após imersão do sujeito em banho (utilizando banheiras, piscinas, spa-pool ou Jacuzzi ${ }^{\circledR}$ ) com circulação de água, durante um período de tempo pré-determinado, seguido por um período de secagem, sem a utilização de toalhas. O número de ciclo de imersão e secagem se repete dependendo da alegação de resistência à água requerida pelo produto, que pode ser considerado como "resistente à água" (40 minutos) ou "muito resistente à água" (80 minutos). A porcentagem de retenção de resistência à água (\%WRR) é determinada pela medição do FPS antes e depois da imersão $(10,12,19)$. 
Although in vivo methods allow simulating conditions closer to reality, they are expensive and require more time for analysis and monitoring. Furthermore, they are in vivo methods performed on healthy subjects. Ethical issues are often discussed, as the volunteers are submitted to conditions of high stress (exposure to UV radiation and long immersion times in baths) (20-23). In contrast, in vitro methods have proven to be interesting alternatives because the cost and time required are lower, the techniques are simple, and they avoid the exposure of healthy volunteers to stress conditions (exposure to UV radiation and long immersion times) (20-25).

Hence, the interest in the development of predictive and validated in vitro methods has been growing. In addition to the benefits previously mentioned, these methods allow for new explorations, as they eliminate the ethical factor of involvement of healthy individuals, and allow for new explorations, such as information on critical wavelength, photostability, and removal of certain ingredients after rinsing. With the in vitro methods, conditions can be conveniently changed, such as water salinity, turbulence, and bath temperature. In addition, an in vitro method allows for rapid comparison of the product profile in the development phase, which cannot yet be applied to humans (20-24). The present work aimed to evaluate the water resistance, by the in vitro method, of a bioactive sunscreen composed of UVA (avobenzone) and UVB (octyl methoxycinnamate) filters, rutin and distinct film/barrier-forming agents (mineral oil, isopropyl myristate and dimethicone), regarding the parameters SPF, critical $\lambda$ and UVA/UVB ratio before/after rinsing, UVA-PF (0).

\section{Material and Methods}

The raw materials and the equipment used in this investigation are described in Table 2 and Table 3, respectively.
Apesar dos métodos in vivo permitirem simular condições mais próximas da realidade, são de alto custo e requerem mais tempo para análise e monitoramento. Ademais, como são métodos in vivo realizados em sujeitos saudáveis, a questão ética é muito discutida, uma vez que os voluntários são submetidos a condições de alto estresse (exposição a radiações UV e longos tempos de imersão em banhos) (20-23). Frente a isso, métodos in vitro mostram-se alternativas interessantes, pois além do custo e tempo requeridos serem inferiores, as técnicas são mais simples, e evita a exposição de indivíduos saudáveis a condições de estresse (exposição a radiações UV e longos tempos de imersão) (20-25).

Diante desse cenário, o interesse no desenvolvimento de métodos in vitro preditivos e validados vem sendo cada vez mais crescente. Além dos benefícios descritos anteriormente, o método elimina o fator ético de envolvimento de indivíduos saudáveis, e permite novas explorações, como informações sobre comprimento de onda crítico, fotoestabilidade e remoção de determinados ingredientes após a lavagem. Com o método in vitro, as condições podem ser convenientemente alteradas, como salinidade da água, turbulência e temperatura do banho. Ademais, o método in vitro permite rápida comparação do perfil de produtos em fase de desenvolvimento, que ainda não podem ser aplicados em seres humanos (2024).

Os estudos apresentados constataram que cada vez mais, a abordagem in vitro vem se tornando alternativa interessante e promissora para a substituição dos ensaios in vivo. Em geral, os resultados obtidos a partir de diversas condições estabelecidas foram satisfatórios e as técnicas foram capazes de reproduzir os ensaios in vivo. Assim sendo, mais estudos de avaliação de resistência à água de fotoprotetores por técnicas in vitro tornam-se fundamentais para consolidar a validade e confiabilidade do método. O presente trabalho teve como objetivo avaliar a resistência à água, por método in vitro, de protetores solares compostos por filtros UVA (avobenzona), UVB (metoxicinamato de octila), rutina e distintos agentes formadores de filme/barreira (óleo mineral, miristato de isopropila e dimeticone), quanto aos parâmetros FPS, $\lambda$ crítico e razão UVA/UVB antes/ depois da lavagem, FP-UVA (0).

\section{Materiais e Métods}

As matérias-primas e os equipamentos utilizados neste estudo estão descritos na Tabela 2 e na Tabela 3, respectivamente. 
Table 2 - Raw materials

Tabela 2 - Matérias-primas

\begin{tabular}{|c|c|c|c|}
\hline $\begin{array}{c}\text { Commercial name / } \\
\text { Nome comercial }\end{array}$ & INCI & $\begin{array}{l}\text { Supplier/ } \\
\text { Fornecedor }\end{array}$ & $\begin{array}{c}\text { Country / } \\
\text { País }\end{array}$ \\
\hline $\begin{array}{l}\text { Citric acid } 10 \% \text { solution / } \\
\text { Ácido cítrico solução a } 10 \%\end{array}$ & Citric acid & IHS Chemical $^{\circledR}$ & England / Inglaterra \\
\hline $\begin{array}{l}\text { Milli-Q ultrapure water / } \\
\text { Água ultrapura Milli-Q }\end{array}$ & Aqua & Merck Milipore $^{\mathbb{R}}$ & $\begin{array}{r}\text { United States / } \\
\text { Estados Unidos }\end{array}$ \\
\hline Avobenzone / Avobenzona & $\begin{array}{c}\text { Butyl } \\
\text { Methoxydibenzoylmethane }\end{array}$ & Volp $^{\mathbb{B}}$ & Brazil / Brasil \\
\hline $\begin{array}{l}\text { Cetearyl alcohol (and) dicetyl phosphate (and) } \\
\text { ethoxylated cetyl alcohol phosphate (10 OE) } \\
\text { (Crodafos TM CES) / Álcool cetearílico (e) fosfato } \\
\text { de dicetila (e) fosfato de álcool cetílico etoxilado } \\
\text { (10 OE) (Crodafos }{ }^{\mathrm{TM}} \text { CES) }\end{array}$ & $\begin{array}{l}\text { Cetearyl Alcohol (and) } \\
\text { Dicetyl Phosphate (and) } \\
\text { Ceteth-10 Phosphate }\end{array}$ & Croda $^{(\mathbb{R}}$ & $\begin{array}{l}\text { United Kingdom / } \\
\text { Reino Unido }\end{array}$ \\
\hline $\begin{array}{c}\text { Co-polymer of sulfonic acid acryloyl- } \\
\text { dimethyltaurate and neutralized vinylpyrrolidone } \\
\text { (Aristoflex }{ }^{\circledR} \text { AVC) / } \\
\text { Co-polímero do ácido sulfônico acriloil- } \\
\text { dimetiltaurato e vinilpirrolidona } \\
\text { neutralizado (Aristoflex }{ }^{\circledR} \text { AVC) }\end{array}$ & $\begin{array}{c}\text { Ammonium } \\
\text { Acryloyldimethyltaurate/VP } \\
\text { Copolymer }\end{array}$ & Clariant $\mathrm{B}^{\circledR}$ & Switzerland / Suíça \\
\hline $\begin{array}{l}\text { 200/350 DC silicone / } \\
\text { Silicone DC } 200 / 350\end{array}$ & Dimethicone & Dow Corning $^{(\mathbb{B})}$ & $\begin{array}{l}\text { United States / } \\
\text { Estados Unidos }\end{array}$ \\
\hline $\begin{array}{l}\text { Phenoxyethanol (and) methylparaben (and) } \\
\text { ethylparaben (and) butylparaben / Fenoxietanol (e) } \\
\text { metilparabeno (e) etilparabeno (e) butilparabeno }\end{array}$ & $\begin{array}{l}\text { Phenoxythenol \& } \\
\text { Methylparaben \& } \\
\text { Ethylparaben \& } \\
\text { Butylparaben \& } \\
\text { Propylparaben }\end{array}$ & Biovital ${ }^{\circledR}$ & $\begin{array}{l}\text { United States / } \\
\text { Estados Unidos }\end{array}$ \\
\hline $\begin{array}{l}\text { Octila methoxycinnamate / } \\
\text { Metoxicinamato de Octila }\end{array}$ & Octyl Methoxycinnamate & PharmaSpecial $^{\circledR}$ & Brazil / Brasil \\
\hline Isopropyl myristate / Miristato de Isopropila & Isopropyl Myristate & PharmaSpecial $^{\circledR}$ & Brazil / Brasil \\
\hline Mineral oil / Óleo Mineral & Paraffinum Liquidum & Volp $^{\circledR}$ & Brazil / Brasil \\
\hline Propylene glycol / Propilenoglicol & Propylene Glycol & All Chemistry ${ }^{\mathbb{B}}$ & Brazil / Brasil \\
\hline Rutin / Rutina & Rutin & Fagron $^{\mathbb{B}}$ & Netherlands / Holanda \\
\hline Triethanolamine / Trietanolamina & Triethanolamine & $\operatorname{Merck}^{\mathbb{B}}$ & $\begin{array}{l}\text { United States / } \\
\text { Estados Unidos }\end{array}$ \\
\hline $\begin{array}{l}\text { Capric and caprylic acid triglycerides / } \\
\text { Triglicérides de acido cáprico e caprílico }\end{array}$ & Caprylic/Capric Trygliceride & Croda $^{(B)}$ & $\begin{array}{l}\text { United Kingdom / } \\
\text { Reino Unido }\end{array}$ \\
\hline
\end{tabular}

Table 3 - Equipment and other

Tabela 3 - Equipamentos e afins

\begin{tabular}{|c|c|c|}
\hline Equipment / Equipamentos & $\begin{array}{l}\text { Brands and models / } \\
\text { Marcas e Modelos }\end{array}$ & Country/ País \\
\hline Mechanical shaker / Agitador mecânico & Fisatom $^{\circledR} 713 \mathrm{D}$ & Brazil /Brasil \\
\hline Analytical balance / Balança analítica & Adventurer OHAUS ${ }^{\circledR}$ & Brazil / Brasil \\
\hline Heating plate / Chapa de aquecimento & $\mathrm{Ika}^{\circledR}$ C-MAG HS7 & Germany / Alemanha \\
\hline $\begin{array}{l}\text { Diffuse reflectance spectrophotometer with } \\
\text { integration sphere / Espectrofotômetro de } \\
\text { reflectância difusa com esfera de integração }\end{array}$ & $\begin{array}{l}\text { Labsphere }{ }^{\mathbb{B}} \text { UV2000S Ultraviolet } \\
\text { Transmittance Analyzer }\end{array}$ & United States / Estados Unidos \\
\hline Refrigerator / Refrigerador & Consul ${ }^{\mathbb{B}}$ Frost Free60 & Brazil / Brasil \\
\hline $\begin{array}{l}\text { pH meter and combined direct immersion electrode } \\
\text { / pHmetro e eletrodo combinado de imersão direta }\end{array}$ & Digimed $^{\circledR}$ DM-22 & Brazil / Brasil \\
\hline $\begin{array}{c}\text { Polymethylmethacrylate (PMMA) plates / Placas } \\
\text { de polimetilmetacrilato (PMMA) }\end{array}$ & HelioScreen ${ }^{\mathbb{B}}$ Helioplate HD6 & United States / Estados Unidos \\
\hline $\begin{array}{l}\text { Milli-Q Simplicity UV water purifier system / } \\
\text { Sistema purificador de água Milli-Q Simplicity UV }\end{array}$ & $\begin{array}{c}\text { Merck Millipore }{ }^{\mathbb{B}} \text { Mili-Q }{ }^{\circledR} \text { Simplicity } \\
\text { UV }\end{array}$ & United States / Estados Unidos \\
\hline Digital thermometer / Termômetro digital & Incoterm $^{\circledR} 6132$ & Brazil / Brasil \\
\hline
\end{tabular}




\section{Preparation of photoprotective formulations}

Three O/W emulsions, each containing different filmforming agents, and a blank emulsion were prepared. The qualitative and quantitative compositions are described in Table 4. The film/barrier agents selected for the water resistance analysis were dimethicone, isopropyl myristate, and mineral oil. The blank formulation corresponded to that which presented the same composition of the three test formulations, without containing a hydrophobic film/barrier agent. The preparation followed the steps described below:

Phase A: The rutin was added to a mixture of water and propylene glycol. The Aristoflex ${ }^{\circledR}$ AVC was hydrated in the resulting mixture using a mechanical stirrer for 10 minutes under agitation of $1000 \mathrm{rpm}$. The $\mathrm{pH}$ value was subsequently adjusted to $9.0 \pm 0.5$ (with triethanolamine), and the system was heated to 70-75 ${ }^{\circ} \mathrm{C}$ on a heating plate.

Phase B: Cetearyl alcohol, dicetyl phosphate, ethoxylated cetyl alcohol phosphate (10 OE), capric and caprylic acid triglycerides, UV filters (avobenzone and octyl methoxycinnamate), and the corresponding film-forming agent / barrier of each formulation were weighed in a beaker and heated to $75^{\circ} \mathrm{C}$.

Maturation process: Phase B was slowly and continously poured into Phase A under $1000 \mathrm{rpm}$ agitation using a mechanical stirrer. Afterwards, the speed was gradually increased to $1500 \mathrm{rpm}$ and maintained for 2 minutes. The preservative was added during stirring. The $\mathrm{pH}$ value of the formulation was adjusted to $6.0 \pm 0.5$ with triethanolamine and/or $10 \% \mathrm{w} / \mathrm{w}$ citric acid solution. The formulations were cooled to room temperature and placed in glass jars with a capacity of $200 \mathrm{~g}$. The formulations were left at room temperature, in the dark, to rest for 48 hours to complete the maturation process and guarantee macroscopic stability.

\section{In vitro SPF efficacy of the formulations}

Three formulations were prepared as $\mathrm{O} / \mathrm{W}$ emulsions. Qualitative and quantitative compositions of the formulations are shown in Table 4. Formulation samples $\left(1.3 \mathrm{mg} / \mathrm{cm}^{2}\right)$ were applied on plates of polymethylmethacrylate (PMMA). Plates were prepared in triplicate to form a uniform film, and dried at room temperature $\left(22{ }^{\circ} \mathrm{C} \pm 1.0\right)$ for 30 minutes in the dark. The absorption spectrum, measured between $250-450 \mathrm{~nm}$, was obtained using a diffuse reflectance spectrophotometer with integration sphere. A glycerin plate was used as blank $(26,27)$. Results were obtained as transmittance versus wavelength curves and

\section{Preparo dos fotoprotetores}

Foram preparadas três emulsões $\mathrm{A} / \mathrm{O}$, cada uma contendo agentes formadores de filme diferentes, e uma emulsão branco. A composição qualitativa e quantitativa de é apresentada na Tabela 4. Os formadores de filme/ barreira selecionados para a análise de resistência à água foram: dimeticone, miristato de isopropila e óleo mineral. A formulação branco correspondeu àquela que apresentou as mesmas composições das três formulações teste, sem conter agente formador de filme/barreira hidrófoba. A preparação das formulações seguiu as etapas descritas a seguir:

Fase A: A rutina foi adicionada à água e ao propilenoglicol e, em seguida, o Aristoflex ${ }^{\circledR}$ AVC foi hidratado no sistema sob agitação de 1000rpm, em agitador mecânico (hélice tipo centrífuga), por 10 minutos. Após, ajustou-se o valor de $\mathrm{pH}$ para $9,0 \pm 0,5$ (trietanolamina) e o sistema foi aquecido a $70-75^{\circ} \mathrm{C}$, em chapa de aquecimento.

Fase B: O Crodafos ${ }^{\circledR}$ CES, triglicérides de ácido cáprico e caprílico, os filtros químicos (avobenzona e metoxicinamato de octila), e o agente formador de filme/barreira correspondente de cada formulação foram pesados em béquer e aquecidos à temperatura de $75^{\circ} \mathrm{C}$, em chapa de aquecimento.

Processo de maturação: A fase B foi vertida lenta e constantemente na fase A, sob agitação de 1000rpm, em agitador mecânico com hélice tipo dentada. Após, a velocidade foi aumentada gradualmente até $1500 \mathrm{rpm}$ e mantida por $2 \mathrm{~min}$. O conservante foi adicionado durante a agitação. $\mathrm{O}$ valor de $\mathrm{pH}$ da formulação foi ajustado para $6,0 \pm 0,5$ com trietanolamina e/ ou ácido cítrico a $10 \% \mathrm{p} / \mathrm{p}$, quando necessário. As formulações foram resfriadas a temperatura ambiente e acondicionadas em potes de vidro com capacidade de $200 \mathrm{~g}$. As formulações foram deixadas em repouso por 48 horas, para a finalização do processo de maturação e garantia de estabilidade macroscópica.

\section{Determinação da eficácia in vitro das formulações}

Foram preparadas três formulações na forma de emulsões O/A, cuja composição qualitativa e quantitativa é apresentada na Tabela 4. As formulações foram aplicadas em placas de PMMA $1,3 \mathrm{mg} / \mathrm{cm}^{2}$ (triplicata) e secas à temperatura ambiente $\left(22^{\circ} \mathrm{C} \pm 1,0\right)$ por 30 minutos no escuro. O espectro de absorção, foi obtido utilizando o espectrofotômetro de reflectância difusa com esfera de integração, entre 250 - $450 \mathrm{~nm}$. Foi utilizada uma placa de glicerina como referência (branco), que representa $100 \%$ de transmissão da luz, 
Table 4 - Qualitative and quantitative composition ( $\% \mathrm{w} / \mathrm{w})$ of bioactive sunscreens

Tabela 4 - Composição qualitativa e quantitativa (\%p/p) das formulações fotoprotetoras.

\begin{tabular}{lccc} 
Components (INCI nomenclature) / & F1 & F2 & F3 \\
Components (Nome INCI) & 3.5 & 3.5 & 3.5 \\
\hline Caprylic/Capric triglyceride & 2.0 & 2.0 & 2.0 \\
Propylene glycol & 6.0 & 6.0 & 6.0 \\
Cetearyl Alcohol (and) Dicetyl Phosphate (and) & 0.5 & 0.5 & 0.5 \\
Ceteth-10 Phosphate & 0.8 & 0.8 & 0.8 \\
Ammonium Acryloyldimethyltaurate/VP Copolymer & 5.0 & 5.0 & 5.0 \\
Phenoxyethanol and methylparaben and & 10.0 & 10.0 & 10.0 \\
ethylparaben, propylparaben and butylparaben & 0.1 & 0.1 & 0.1 \\
Butyl metoxydibenzoylmethane & - & - & 3.0 \\
Octyl methoxycinnamate & - & 3.0 & - \\
Rutin & 3.0 & - & - \\
Mineral oil/ Paraffinum Liquidum & 100 & 100 & 100 \\
Isopropyl myristate & & \\
200/350 DC silicone/ Dimethicone & & \\
Purified water q.s.p. & * quantity necessary to reach 100\%; (-) raw material not added / & & \\
* quantidade suficiente para 100\%; (-) matéria-prima não adicionada &
\end{tabular}

represented the average of the nine points transmittance curves of the PMMA plates. With the measurement, values of SPF, critical $\lambda$, UVA/UVB ratio, and UVA$\mathrm{PF}(0)$ were obtained (27).

In vitro water resistance assessment of the bioactive formulations

Bioactive formulations were subjected to an in vitro water resistance test, based on the test recommended by COLIPA (27), and adapted from the method validated by Ahn and collaborators (20). After absorption profile determination, the plates (triplicate) were immersed in a water bath, held in clips, at $42.0 \pm 1.0{ }^{\circ} \mathrm{C}$. After agitation at $150 \mathrm{rpm}$ for 60 minutes, the plates were removed and freely dried at room temperature $(23.0 \pm$ $2.0^{\circ} \mathrm{C}$ ) for 30 minutes, protected from light (20).

\section{Determination of percent water resistance retention}

Water resistance capacity of the sunscreens, in percentage of Water Resistance Retention (\% WRR), was calculated according to Equation 1 (10).

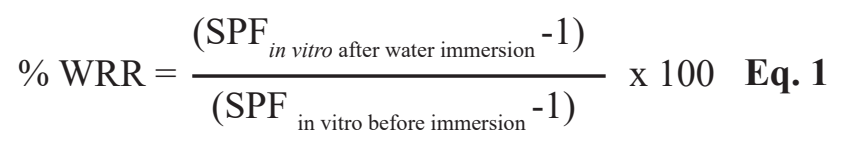

antes da leitura $(26,27)$. Os resultados de absorção foram obtidos em forma de curvas de transmitância versus comprimento de onda e representaram a média das curvas de transmitância de nove pontos das placas de PMMA. Com a medição, foram obtidos valores de FPS, $\lambda$ crítico, razão UVA/UVB e FP-UVA (0) (27).

Avaliação de resistência à água in vitro das formulações bioativas

As formulações fotoprotetoras foram submetidas ao teste de resistência à água in vitro, baseado no teste preconizado pela COLIPA (27), e adaptado do método validado por Ahn e colaboradores (20). Após a medição do perfil de absorção, as placas (triplicata) foram mergulhadas em banho com clips, sob agitação de 150 rpm a uma temperatura controlada de $42^{\circ} \mathrm{C} \pm 1,0$. Após um período de lavagem de 60 minutos, as placas foram retiradas e secas à temperatura ambiente $\left(23,0 \pm 2,0^{\circ} \mathrm{C}\right)$ por 30 minutos, protegidas da luz (20).

Determinação de porcentagem de Retenção de Resistência à Água

Para a avaliação de capacidade de resistência ao enxágue de um fotoprotetor, realizou-se o cálculo da porcentagem de Retenção de Resistência à Água (\%WRR) de acordo com a Equação 1 (10).

$\% \mathrm{WRR}=\frac{\left(\mathrm{FPS}_{\text {in vitro após a imersão em áqua }}{ }^{-1}\right)}{\left(\mathrm{FPS}_{\text {in vitro antes da imersão }}-1\right)} \times 100$ Eq. 1 
The $\%$ WRR must be greater than $50 \%$ for a sun product to be considered water resistant after a certain water immersion time (10).

\section{Statistical analysis}

The results were performed using IBM $^{\circledR}$ SPSS $^{\circledR}$ Statistics 22 (IBM Corp., Armonk, NY, USA) program. Statistical differences were verified for the mean values $(n=3)$ of the efficacy parameters (FPS, critical $\lambda$, UVAPF (0) and UVA/UVB ratio) and \% WRR. The ANOVA was used, followed by post hoc testing, when necessary.

\section{Results}

After the resting period (48 hours), all formulations presented physical stability, as no phase separation was observed. In vitro SPF values of each formulation were obtained before and after rinsing with water (Figure 1A). Samples containing mineral oil and isopropyl myristate showed lower in vitro SPF values than the formulation with dimethicone. A difference between the SPF values before and after rinsing in the samples $(p=0.001)$ was observed. The sample with dimethicone showed higher SPF after rinsing when compared with isopropyl myristate and mineral oil $(\mathrm{p}=0.001)$. The last two samples did not show differences concerning the SPF variation after rinsing with water, both demonstrating a similar performance for the SPF maintenance in a water resistance test. Two-way ANOVA interaction analysis (water immersion/formulation) demonstrated that there was a difference between the formulations $(p=0.003)$.
O produto fotoprotetor, para ser considerado resistente à água, deve apresentar seu \%WRR à 50\%, após determinado tempo de lavagem (10).

\section{Análise estatística}

A análise estatística dos resultados foi realizada no programa IBM $^{\circledR}$ SPSS $^{\circledR}$ Statistics 22 (IPM Corp., Armonk, NY, USA). Para verificar as diferenças estatísticas dos valores médios $(n=3)$ das análises de eficácia (FPS, $\lambda$ crítico, FP-UVA (0) e razão UVA/ UVB) e \%WRR. ANOVA foi utilizada, seguida de teste de post hoc, quando necessário.

\section{Resultados}

Após o período de repouso (48 horas), todas as formulações apresentaram estabilidade física, não sendo observada separação de fases. Foram obtidos os valores de FPS in vitro de cada formulação antes e após a lavagem com água (Figura 1A). As formulações contendo óleo mineral e miristato de isopropila apresentaram valores de FPS in vitro inicial menores que a formulação com dimeticone. Observou-se que, houve diferença entre os valores de FPS antes e após lavagem nas formulações $(p=0,001)$. A formulação com dimeticone apresentou FPS maior pós-lavagem quando comparada à amostra com miristato de isopropila e com óleo mineral $(p=0,001)$. As duas últimas formulações não demonstraram diferenças entre si em relação à variação de FPS após a lavagem com água, tendo ambas desempenho similar para a manutenção de FPS
A

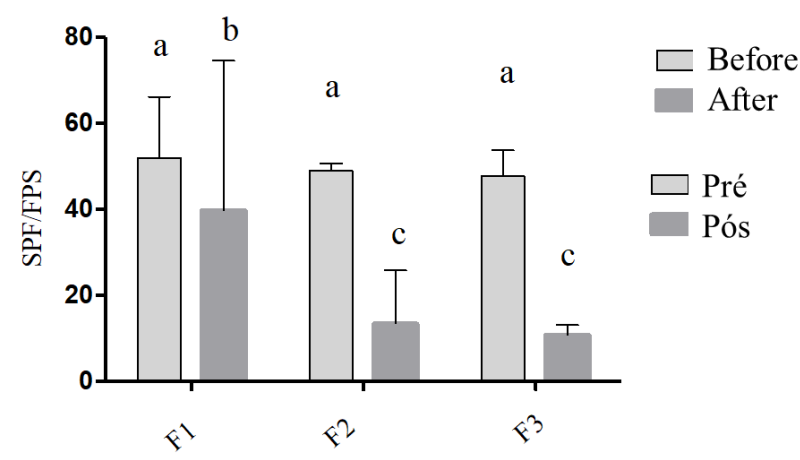

B

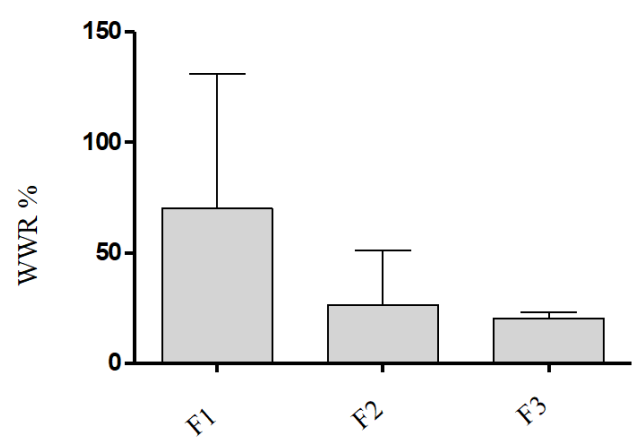

Figure 1 - Sun protection factor (SPF) before and after rinsing with water (A). Different letters over the columns show differences among the samples. Water Resistance Retention Percentage (\% WRR) according to film / barrier formers after rinsing with water (B).

Figura 1 - Fator de proteção solar (FPS) antes e após a lavagem com água (A). Letras diferentes sobre as colunas demonstram diferenças entre as formulações. Porcentagem de Retenção de Resistência à Água (\%WRR) de acordo com os formadores de filme/barreira após a lavagem com água (B). 

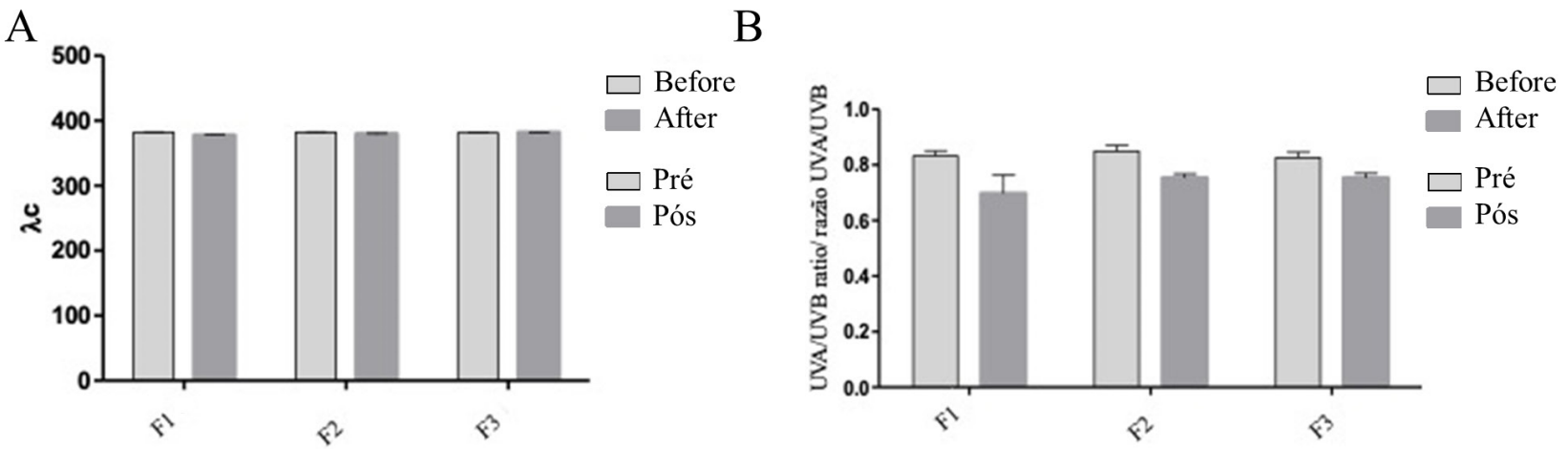

Figure 2 - Critical wavelength $(\lambda c)(A)$ and UVA / UVB ratio (B) according to the film / barrier formers, before and after rinsing with water.

Figura 2 - Comprimento de onda crítico $(\lambda c)(A)$ e Razão UVA/UVB (B) de acordo com os formadores de filme/barreira, antes e após a lavagem com água.

The mean \% WRR values (Equation 1) are illustrated in Figure 1B. Only the dimethicone sample obtained the requirement (to be considered water resistant) of $\%$ WRR greater than $50 \%(10)$.

The average values of $\lambda \mathrm{c}$ are shown in Figure 2A. The formulations showed similar values regarding the variation of this parameter after the water resistance test. The average values of the UVA/UVB ratio are illustrated in Figure 2B. The difference was observed before and after rinsing with water in the formulations $(\mathrm{p}<0.01)$. However, the samples were similar in terms of the variation of this parameter after the water resistance test $(p=0.155)$. By ANOVA analysis (water immersion and formulation), there were no differences among samples $(\mathrm{p}=0.256)$.

The UVA protection was also evaluated before water immersion by mean values of UVA-PF(0) (Figure 3 ). No significant difference in this parameter was observed among the samples studied $(\mathrm{p}=0.083)$ concerning the UVA-PF(0) attribution. em ensaio de resistência à água. A análise de interação por ANOVA de dois fatores (enxágue/formulação) demonstrou que houve diferença entre as formulações $(p=0,003)$.

A partir dos valores de FPS in vitro, foi realizado o cálculo de \%WRR para cada formulação utilizando a Equação 1. Os valores médios de \%WRR são ilustrados na Figura 1B. Ao comparar o FPS in vitro em seus valores brutos, observou-se que a única formulação que atendeu ao requisito de \%WRR maior que $50 \%$, foi o dimeticone, portanto, considerada formulação resistente à água (10).

Os valores médios de $\lambda c$ são apresentados na Figura 2A. As formulações apresentaram valores similares quanto à variação desse parâmetro antes e após o ensaio de resistência à água $(\mathrm{p}=0,117)$. Os valores médios de razão UVA/UVB são ilustrados na Figura 2B. Foi observada diferença antes e após a lavagem com água nas formulações $(p<0,01)$. No entanto, os fotoprotetores foram similares quanto à variação desse parâmetro após o ensaio de resistência à água $(p=0,155)$. Na análise por ANOVA de dois fatores (tempo e formulação) não houve diferença entre as amostras $(p=0,256)$.

O fator de proteção contra UVA foi avaliado antes da imersão pela média de FP-UVA (0) (Figura 3). Observou-se que não houve diferença neste parâmetro entre as amostras $(p=0.083)$ com relação ao fator de proteção UVA antes da imersão. 


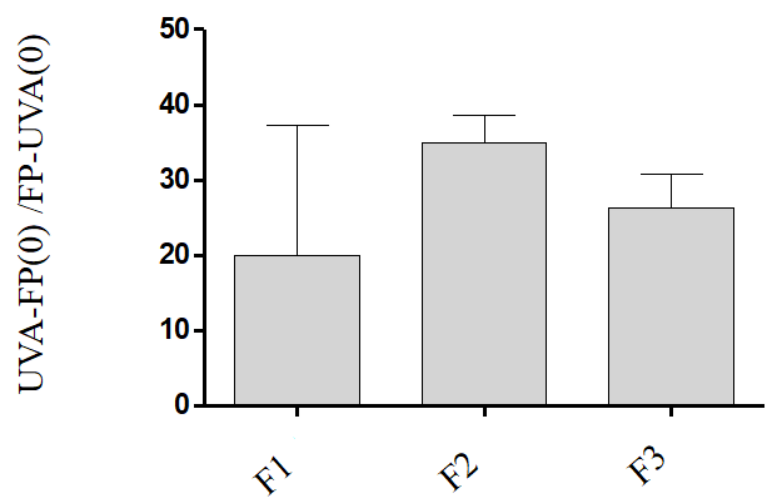

Figure 3 - UVA protection factor before rinsing (FP-UVA (0)), according to the film / barrier formers. Figura 3 - Fator de proteção UVA antes da immersão (FP-UVA (0)), de acordo com os formadores de filme/ barreira.

\section{Discussion}

The bioactive sunscreen sample used in this investigation used rutin as an efficacy booster, providing the product with not only a better SPF profile but also antioxidant properties $(4,5)$. Investigations regarding the use and development of in vitro water resistance tests have been proposed. Ahn et al. (20) developed an in vitro method by changing test parameters (immersion time and number of revolutions per minute). The best responses were obtained under the conditions of 140 $\mathrm{rpm} / 75 \mathrm{~min}$ and $150 \mathrm{rpm} / 60 \mathrm{~min}$, the latter being selected for validation, due to the shorter time. The in vitro results of \% WRR showed a lower coefficient of variation $(14.1 \%)$ compared to in vivo tests $(28.0 \%)$. In addition, the in vitro test was able to reflect changes in the concentration of water-resistant ingredients, thus being a method capable of reproducing the results of in vivo tests.

In order to develop in vitro test methods for water resistance of photoprotectors, Choquenet et al. (21) carried out tests based on the conditions determined from in vivo assays. A pre-test was performed in three different conditions: stagnant distilled water bath, agitated distilled water bath and agitated saline water bath. The results showed that the second condition presented a more critical result, but with results consistent with the parameters considered acceptable for water resistance.

Pissavini et al. (24) proposed rinsing resistance tests with different water quality (tap water, saline water, and distilled water) and increasing immersion times (5, 20, and 40 minutes). According to the study, distilled water

\section{Discussão}

O fotoprotetor bioativo usado nesta pesquisa empregou a rutina como substância para elevar a eficácia das amostras, doando ao produto não apenas melhor FPS, como, também, propriedade antioxidante $(4,5)$. Investigações diversas foram propostas para o uso e desenvolvimento de testes in vitro de resistência à água. Ahn e colaboradores (20) desenvolveram um método in vitro por meio de alteração de parâmetros de ensaio (tempo de imersão e número de revoluções por minuto). As melhores repostas foram obtidas nas condições de $140 \mathrm{rpm} / 75 \mathrm{~min}$ e $150 \mathrm{rpm} / 60 \mathrm{~min}$. Os resultados in vitro de \%WRR apresentaram coeficiente de variação menor $(14,1 \%)$ comparado aos testes in vivo $(28,0 \%)$. Ainda, o teste in vitro foi capaz de refletir às mudanças de concentração de ingredientes resistentes à água, sendo assim um método capaz de reproduzir os resultados dos testes in vivo.

Choquenet e colaboradores (21), com o intuito de desenvolver métodos de teste in vitro de resistência à água de fotoprotetores, realizaram ensaios baseados nas condições determinados em ensaios in vivo. Préensaio foi realizado em três condições diferentes: banho de água destilada estagnada, banho de água destilada com agitação e banho de água salina com agitação. Os resultados apontaram que a segunda condição apresentou resultado mais crítico, porém com resultados coerentes com os parâmetros considerados aceitáveis de resistência à água.

Pissavini e colaboradores (24) propuseram testes de resistência ao enxágue com qualidade de água diferentes (água de torneira, água salina e água destilada) e tempos 
showed the best results for resistance to rinsing. The conductivity of this is one of the factors that influences the water resistance capacity of a product, and this relationship occurs in an inverse way. In this study, ten photoprotective products were evaluated, in increasing immersion times, to obtain visualization of the decay kinetics of \% WRR over time. The \% WRR of in vitro assays were correlated to in vivo assays, and the results showed significant $(R)$ regression value $(0.92$, 0.83 , and 0.78 for 5,20 , and 40 minutes of immersion, respectively) and slopes near to $1(1.28,1.19$ and 1.12 for 5, 20 and 40 minutes of immersion), which indicated a good correlation between the two methodologies.

Stokes and collaborators (22) utilized a different approach for in vitro evaluation of water resistance. In contrast to the previously mentioned studies, which used PMMA plates as a substrate, excisions of human epidermis were used. Aliquot of $2.0 \mathrm{mg} / \mathrm{cm}^{2}$ of formulation was applied to the excisions, which were immersed in a water bath in two series of 20 minutes each under constant agitation and a temperature of $25 \pm$ $2{ }^{\circ} \mathrm{C}$. The SPF was obtained before and after immersion in a bath. The technique, previously validated, showed that the SPFs of the formulations obtained before and after immersion in water showed no differences in relation to the SPFs obtained in vivo. The results were considered promising for the development of reliable and reproducible in vitro methods.

Components capable of forming films are added for a sunscreen to enable water resistance. Film-formers include oils, polymers, and copolymers, esters, and silicones. Their characteristics provide resistance to rinsing, which increases the ability to photoprotect against UV radiation. Likewise, some compounds can provide positive effects on the sensory behavior of the formulation, a property known mainly in silicones. $(11,28)$.

Figure 1a demonstrates that the formulation containing dimethicone showed a higher SPF before and after rinsing when compared to samples of mineral oil and isopropyl myristate, suggesting an effect of greater water resistance. Furthermore, it was observed that the dimethicone sample met the requirement of \% WRR greater than 50\% (Equation 1 and Figure 1b); therefore, it can be considered a water-resistant formulation (10).

Dimethicone, a type of silicone, demonstrated a waterresistant formulation, and this result converges with the property of silicones forming water-resistant films. Silicones are able of forming homogeneous polymeric films, this ensuring the substantivity (adhesion, water de imersão crescentes (5, 20 e 40 minutos). Segundo o estudo, a água destilada apresentou os melhores resultados de resistência à lavagem. A condutividade desta é um dos fatores que influencia na capacidade de resistência a água de um produto, sendo que essa relação ocorre de maneira inversa. Nesse estudo, dez produtos fotoprotetores foram avaliados, em tempos de imersão crescentes, para obter visualização da cinética de decaimento do \%WRR com o tempo. As \%WRR dos ensaios in vitro foram correlacionadas aos ensaios in vivo, e os resultados demonstraram valor de regressão (R) significativo $(0,92,0,83$ e 0,78 para 5,20 e 40 minutos de imersão) e slope próximos a $1(1,28,1,19$ e 1,12 para 5, 20 e 40 minutos de imersão), o que indicou boa correlação entre as duas metodologias.

Stokes e colaboradores (22) realizaram abordagem diferente para avaliação in vitro de resistência à água. Em contrate aos estudos previamente citados, que utilizaram placas de PMMA como substrato, foram utilizadas excisões de epidermes humanas. Alíquota de $2,0 \mathrm{mg} / \mathrm{cm}^{2}$ de formulação foi aplicada nas excisões, que foram imersas em banho de água em duas séries de 20 minutos, sob agitação constante e temperatura de $25 \pm 2{ }^{\circ} \mathrm{C}$. O FPS foi obtido antes e após a imersão em banho. A técnica, previamente validada, demonstrou que os FPS's das formulações obtidos antes e após a imersão em água não apresentaram diferenças em relação aos FPS's obtidos in vivo. Os resultados foram considerados promissores para o desenvolvimento de métodos in vitro confiáveis e reprodutíveis (24).

Para um protetor solar adquirir a propriedade de resistência à água, são adicionados componentes capazes de formar filmes. Os formadores de filme incluem óleos, polímeros e copolímeros, ésteres e silicones, e sua característica confere resistência a lavagem o que aumenta a sua capacidade de fotoproteção contra radiação UV. Ademais, alguns compostos podem proporcionar efeitos positivos no comportamento sensorial da formulação, propriedade conhecida principalmente nos silicones $(11,28)$.

Conforme observado na Figura 1a, a formulação contendo dimeticone apresentou um FPS maior antes e após lavagem quando comparado com as amostras de óleo mineral e miristato de isopropila, sugerindo um efeito de maior resistência a água. Ademais, observouse que a única formulação que atendeu ao requisito de \%WRR maior que 50\% (Equação 1 e Figura 1b), foi o dimeticone, portanto, considerada formulação resistente à água (10). Tal resultado pode ser resultante da propriedade de silicones formarem filmes resistentes à água. Os silicones são capazes de formar filmes 
resistance) of the sunscreens on the skin. Additionally, its hydrophobic property prevents water from contacting the filters, minimizing their removal during rinsing $(26,28)$.

Although mineral oil and isopropyl myristate can form occlusive and hydrophobic barriers, due to their emollient properties, they were not efficient enough compared to the dimethicone. In addition, the silicone materials could show the property of skin adhesion, which could favor water resistance (28).

The sunscreen water resistance uses to consider only the photoprotective activity against UVB, thus, the evaluation of protection against UVA is important to ensure that water resistant sunscreens offer protection across the UV spectrum (21). The evaluation regarding protection against UVA was determined from the following parameters: $\lambda c$, UVA/UVB ratio, and UVA$\mathrm{PF}(0)$ before rinsing.

The $\lambda \mathrm{c}$ values did not show any difference among the samples and after rinsing. This result demonstrated that none of the samples interfered with this parameter, which considers that a photoprotector must have a value of $\lambda c$ greater than $370 \mathrm{~nm}$ (Figure 2a) in order to be indicated as providing adequate protection against UVA damage $(19,27)$. However, a difference was observed in the UVA/UVB ratio of the samples before and after rinsing with water $(\mathrm{p}<0.001)$. Furthermore, the samples showed similar results after water rinsing $(p=0.256)$. It was observed that there was no difference in UVA-PF(0) parameter among the samples studied before immersion (Figure 3), indicating that the film/ barrier agents did not show differences $(p=0.083)$ concerning the UVA-PF(0).

\section{Conclusion}

The presence of dimethicone provided less variation in the in vitro SPF value after water immersion when compared to isopropyl myristate and mineral oil. The determination of the percentage of water resistance demonstrated that the sample with dimethicone was considered water resistant, while myristate of isopropyl and mineral oil showed similar average values of water resistance, but lower than recommended values. The analysis of $\lambda c, U V A / U V B$ ratio, and UVA-PF $(0)$ among the formulations indicated that the film/barrier agents showed the same performance in maintaining these parameters. homogêneos, garantindo substantividade (adesão, resistência à agua) dos filtros na pele. Ademais, sua propriedade hidrófoba impede o contato da água com os filtros, minimizando a remoção destes durante a lavagem $(26,28)$.

Apesar do óleo mineral e do miristato de isopropila serem capazes de formar barreiras oclusivas, e hidrofóbicas, por suas propriedades emolientes, as mesmas não foram suficientemente eficientes, provavelmente, pela ausência de filme, cuja propriedade "wash-off" é bem estabelecida para silicones. Ademais, os silicones apresentam propriedades de adesão à pele, o que reforça esse efeito (28).

A propriedade de resistência à água, usualmente, é definida pela medição da atividade fotoprotetora contra UVB. Sendo assim, a avaliação da proteção contra UVA demonstra-se importante para garantir que o protetor solar resistente à água ofereça proteção em todo o espectro UV (21). A avaliação contra radiação UVA foi determinada pelos seguintes parâmetros: $\lambda c$, razão UVA/UVB e FP-UVA(0) antes do banho.

Valores de $\lambda \mathrm{c}$ não apresentaram diferenças entre as amostras e após o enxágue. Tal resultado indicou que nenhuma das amostras sofreu interferência neste parâmetro, que considera que o fotoprotetor necessita ter valor de $\lambda$ c superior a $370 \mathrm{~nm}$ (Figura 2a) para ser considerado de proteção de amplo espectro e contra danos provocados pelo UVA. No entanto, diferença foi observada na razão UVA/UVB das amostras antes e depois do banho $(p=0,001)$, porém, os resultados foram considerados similares após o enxágue $(\mathrm{p}=0,256)$. Os valores médios de FP-UVA (0) são ilustrados na Figura 3. Observou-se que não houve diferença desse parâmetro entre as formulações estudadas, indicando que os formadores de filme/barreira não apresentaram diferenças $(\mathrm{p}=0,083)$ com relação à atribuição de fator de proteção UVA antes da irradiação.

\section{Conclusão}

A presença do dimeticone proporcionou menor variação de FPS após a lavagem com água, quando comparado ao miristato de isopropila e ao óleo mineral A determinação da porcentagem de resistência à água demonstrou que a formulação com dimeticone foi considerada resistente à água, enquanto o miristato de isopropila e óleo mineral apresentaram valores médios de resistência à agua próximos entre si, mas inferiores ao preconizado como "resistente à água." As análises de $\lambda \mathrm{c}$, razão UVA/UVB, 


\section{Acknowledgments}

The authors would like to express their thanks to National Council for Scientific and Technological Development (CNPq) Process 305250 / 2019-1; Coordination for the Improvement of Higher Education Personnel Brazil (CAPES) for financial code 001 and São Paulo State Research Support Foundation (FAPESP, Process 2015/11194-6 and 2016/22000-0) for financial support.

\section{Author Contributions}

CYS, conceptualization, experimental implementation, data analysis and drafting; ALMJ and FVL, data analysis, drafting and reviewing; GAM, WLAR, CACJ and ERAR, drafting and editing; CR and MVRV, editing and reviewing; ARB, conceptualization, supervision, final redaction.

\section{Conflict of interest}

The authors declare no conflict of interest. The senior editors co-authoring this manuscript had no participation in the review nor in the decision process.

All authors have declared there were no financial and/ or personal relationships that may present a potential conflict of interest. e FP-UVA(0) entre as formulações indicaram que os formadores de filme/barreira apresentaram o mesmo desempenho na manutenção desses parâmetros.

\section{Agradecimentos}

Os autores agradecem o Conselho Nacional de Desenvolvimento Científico e Tecnológico (CNPq) Process 305250/2019-1; Coordenação de Aperfeiçoamento de Pessoal de Nível Superior - Brasil (CAPES) código financeiro 001 e Fundação de Amparo a Pesquisa do Estado de São Paulo (FAPESP, Processo 2015/11194-6 e 2016/22000-0) pelo apoio financeiro.

\section{Contribuição dos Autores}

CYS, concepção do estudo, experimento, análise de dados e redação; ALMJ e FVL, análise de dados, redação e revisão; GAM, WLAR, CACJ e ERAR, redação e edição; CR e MVRV, edição e revisão; ARB, concepção do estudo, supervisão, redação final.

\section{Conflito de interesse}

Os editores senior envolvido na autoria deste manuscrito não teveram qualquer participação no processo de revisão ou de decisão.

Todos os autores declararam não haver relações financeiras e/ou pessoais que possam representar um potencial conflito de interesses. 


\section{References / Referências}

1. Balogh TS, Velasco VRM, Pedriali CA, Kaneko TM, Baby AR. Proteção à radiação ultravioleta : recursos disponíveis na atualidade em fotoproteção. An Bras Dermatol. 2011;86(4):732-742.

2. Zhang LW, Al-Suwayeh SA, Hsieh PW, Fang JY. A comparison of skin delivery of ferulic acid and its derivatives: Evaluation of their efficacy and safety. Int J Pharm. 2010;399(1-2):44-51. doi:10.1016/j.ijpharm.2010.07.054

3. Reyes E, Vitale MA. Advances in photoprotection. Molecular mechanisms. Piel. 2013;28(4):235-247. doi:10.1016/j.piel.2012.04.011

4. Peres DA, de Oliveira CA, da Costa MS, et al. Rutin increases critical wavelength of systems containing a single UV filter and with good skin compatibility. Ski Res Technol. 2016;22(3):325-333. doi:10.1111/srt.12265

5. Morocho-Jácome AL, Freire TB, Oliveira AC, et al. In vivo SPF from multifunctional sunscreen systems developed with natural compounds - a review. J Cosmet Dermatol. doi:10.1111/jocd.13609

6. Souza FP, Campos GR, Packer JF. Determinação da atividade fotoprotetora e antioxidante em emulsões contendo extrato de Malpighia glabra L. - Acerola. Rev Ciências Farm Básica e Apl. 2013;34(1):69-77. doi:10.11693/hyhz20181000233

7. De Oliveira CA, Peres DDa, Rugno CM, et al. Functional photostability and cutaneous compatibility of bioactive UVA sun care products. J Photochem Photobiol B Biol. 2015;148:154-159. doi:10.1016/j.jphotobiol.2015.04.007

8. Velasco MVR, Balogh TS, Pedriali CA, et al. Associação da Rutina com p-Metoxicinamato de Octila e Benzofenona-3: Avaliação In Vitro da Eficácia Fotoprotetora por Espectrofotometria de Refletância. Lat Am J Pharm. 2008;27(1):23-27. doi:http://hdl.handle. net/10915/7576

9. Trotta V, Goios F, Monteiro H, Almeida IF, Scalia S. Influence of lipid microparticle encapsulation on in vitro efficacy, photostability and water resistance of the sunscreen agents, octyl methoxycinnamate and butyl methoxydibenzoylmethane. Drug Dev Ind Pharm. 2014;40(9):1233-1239. doi:10.3109/03639045.2013.814062

10. Cosmetics Europe: Guidelines for Evaluating Sun Product Water.; 2005.

11. American S. What Makes a Sunscreen Water-Resistant ? Vol 6.; 2013.

12. Rigano L. "Very" Water-resistant Sunscreens. Cosmet Toilet. 2014; $\operatorname{march}(20): 1-7$.

13. Milesi SS, Guterres SS. Fatores determinantes da eficácia de fotoprotetores. Cad Farmácia. 2002;18(2):81-87.

14. Cabral LDS, Pereira SO, Partata A. Filtros solares e Fotoprotetores mais utilizados nas formulações no Brasil. Rev Científica do ITPAC. 2011;4(3):732-742.

15. Felton LA. Mechanisms of polymeric film formation. Int J Pharm. 2013;457(2):423-427. doi:10.1016/j.ijpharm.2012.12.027

16. Kathe K, Kathpalia H. Film forming systems for topical and transdermal drug delivery. Asian J Pharm Sci. 2017;12(6):487-497. doi:10.1016/j.ajps.2017.07.004

17. Weber TM, Schoelermann AM, Breitenbach U, Scherdin U, Kowcz A. Hand and foot moisturizers. In: Draelos ZD, ed. Cosmetic Dermatology: Products and Procedures. 1st ed. Singapure: Wiley-Blackwell; 2010:131-133. doi:10.1002/9781444317657.ch35

18. Hunter A, Trevino M. Film formers enhance water resistance and SPF in sun care products. Cosmet Toilet. 2004;119(7):51-56.

19. Guidance for Industry Labeling and Effectiveness Testing : Sunscreen Drug Products for Over-The-Counter Human Use - Small Entity Compliance Guide. New Hampshire: Food and Drug Administration; 2012.

20. Ahn S, Yang H, Lee H, Moon S, Chang I. Alternative evaluation method in vitro for the water-resistant effect of sunscreen products. Ski Res Technol. 2008;14(2):187-191. doi:10.1111/j.1600-0846.2007.00276.x

21. Choquenet B, Couteau C, Paparis E, Coiffard LJM. Development of an in vitro test to determine the water-resistance of sunscreens. Pharmazie. 2008;63(7):525-527. doi:10.1691/ph.2008.7404

22. Stokes RP, Diffey BL, Dawson LC, Barton SP. A novel in vitro technique for measuring the water resistance of sunscreens. Int J Cosmet Sci. 1998;20(4):235-240. doi:10.1046/j.1467-2494.1998.176609.x

23. Cheignon C, Couteau C, Billon-Chabaud A, Marques Da Silva E, Paparis E, Coiffard LJM. Comparison of two in vitro methods to evaluate the water resistance of sunscreens. Pharmazie. 2012;67(2):116-119. doi:10.1691/ph.2012.1048

24. Pissavini M, Alard V, Heinrich U, et al. In vitro assessment of water resistance of sun care products: A reproducible and optimized in vitro test method. Int J Cosmet Sci. 2007;29(6):451-460. doi:10.1111/j.1468-2494.2007.00407.x

25. Sohn M, Malburet C, Caliskan G, et al. In vitro water resistance testing using SPF simulation based on spectroscopic analysis of rinsed sunscreens. Int J Cosmet Sci. 2018;40:217-235.

26. Moraes CAP, Arêas EPG, Velasco MVR. Assessment of functional stability of photoprotective formulations containing rutin succinate. Cosmetics. 2017;4(3):1-14. doi:10.3390/cosmetics4030027

27. COLIPA. In Vitro Method for the Determination of the UVA Protection Factor and "Critical Wavelength" Values of Sunscreen Products. European Cosmetic, Toiletry and Perfumery Association; 2011.

28. Klykken P, Servinski M, Thomas X. Silicone Film-Forming Technologies for Health Care Applications. Dow Corning Corp Form. 2004;52-1068A-0:1-7. 